\section{The search of antifragile architecture}

PALABRAS CLAVE | FRAGILIDAD | ROBUSTEZ ANTIFRAGILIDAD

KEYWORDS | FRAGILITY | ROBUSTNESS | ANTIFRAGILITY

\section{| RESUMEN |}

La investigación realizada consistió en extrapolar la teoría de la antifragilidad forjada en el ámbito de la economía hacia la esfera de la arquitectura con el objetivo de proponer una vía evolutiva para poder enfrentar la complejidad característica de la realidad contemporánea. La hipótesis postulaba la existencia de la antifragilidad en arquitectura a través de un comportamiento evolutivo de las construcciones tras enfrentarse a una crisis. Se seleccionaron cuatro ejemplos y se definieron cuatro criterios para poder analizarlos. Se llegaron a conclusiones parciales de cada criterio, se catalogaron los ejemplos como frágiles, robustos o antifrágiles y, se apoyó el análisis con imágenes de las obras y propuestas analizadas. Se llegó a la conclusión de que no es posible un comportamiento evolutivo sin la intervención del hombre, por lo que es responsabilidad de su ingenio otorgarle antifragilidad a los sistemas arquitectónicos.

\section{| ABSTRACT |}

The investigation consisted on extrapolate the theory of antifragility forged in the field of the economy towards the sphere of architecture in order to propose an evolutionary path to be able to face the complexity of the contemporary reality. The hypothesis of the investigation defined that it was possible the existence of antifragility in architecture through an evolutionary behave in the buildings after face a crisis. Four examples were selected and four criteria were defined to implement the analysis. The collected information was recorded in thematic cards and once completed the stage of reading and analyzing the literature; four theoretical criteria were defined to perform analysis that sought to detect antifragility in those examples. Partial conclusions were reached, the examples were catalogue in fragile, rugged or antifragile and, the analysis was supported by images of the work and proposals of the examples. The finat conclusion was that evolutionary behavior is not possible without human intervention, and, as a result, it is the responsibility of human to give antifragility to architectonics systems by using human ingenuity.

\title{
La búsqueda de la arquitectura antifrágil ${ }^{*}$
}

\author{
KATIA MUNJIN PAIVA***Santiago, Chile·kmunjin@gmail.com
}

\begin{abstract}
EI trabajo realizado en el Seminario de — Investigación se fundamentó en la postura personal de que el proceso de titulación es una instancia que debe ser entendida como algo continuo y como una oportunidad para el estudiante de proponer y aportar en el ámbito de la arquitectura. Se tomó la decisión de iniciar con el seminario -ya que estaba la posibilidad de iniciar con la práctica- porque se quiso elaborar una teoría sobre lo que se enfrentaría posteriormente en la Práctica Profesional Externa y el Proyecto de Título. Además de cumplir con la exigencia académica, entendiendo que como instancias curriculares es obligación para el estudiante cursar cada una de ellas, se quiso potenciar aquello con el desarrollo de un nuevo paradigma para la esfera teórica de la arquitectura aplicando el concepto antifrágil y estableciendo pautas y criterios de cómo ese paradigma se podría trasladar a un nivel más práctico que se relacionara directamente con el quehacer del ejercicio profesional.
\end{abstract}

La antifragilidad es un concepto del cual se tuvo conocimiento en una experiencia de intercambio en la ciudad de Turín, Italia, el año 2014. En aquel lugar, junto con un grupo de estudiantes de un taller de restauración arquitectónica cursado en el marco del intercambio, se estudió la posibilidad de aplicar este concepto en arquitectura, específicamente en un proyecto de rehabilitación y ampliación de un edificio patrimonial.

El concepto antifrágil nace como respuesta a una serie de fenómenos que el economista Nassim Nicholas Taleb -creador del conceptojunto con otros autores de diversas áreas, identificaron durante los últimos cinco años. Estos fenómenos, se manifiestan ahora como consecuencia de una serie de procesos sociales, históricos, económicos, tecnológicos y culturales que han sucedido durante el siglo XX y los años transcurridos del presente siglo. Algunos de esos fenómenos -como el exceso de información, la imprevisibilidad de los sucesos y la complejidad de las relaciones- se relacionan con la percepción caótica que se tiene hoy en día de la realidad y la sensación de ingobernabilidad que tiene el hombre sobre los sucesos que irrumpen en ella. La cantidad de variables involucradas en ella y la velocidad con que se desarrollan, han impedido que el hombre pueda gobernarla y, de todas formas, él ha intentado tener el control de lo incontrolable, y con ello, ha generado una serie de contradicciones e incoherencias en el

\footnotetext{
* Artículo en base a los resultados del Seminario de investigación "Antifragilidad en la Arquitectura. Sistema orgánico para resistir el desorden y evolucionar", realizado por la autora y guiada por el profesor Max Aguirre González, en el Instituto de Historia y Patrimonio de la Universidad de Chile, durante el semestre de otoño de 2015.

** Licenciada en Arquitectura (2014) de la Facultad de Arquitectura y Urbanismo de la Universidad de Chile.
} 
quehacer de diversas prácticas dentro de esta realidad.

El hombre ahora es un extraño de su pasado e ignorante de su futuro, al intentar tener el control de todo, ha creado barreras y obstáculos para poder ver con claridad los componentes de la realidad que debe enfrentar. Ante esas dificultades, la realidad se presenta ante el hombre envuelta por un manto de opacidad, que oculta aquello que no se ve a simple vista y, aquello que sí se puede ver no tiene la claridad suficiente para que el hombre pueda trabajar, así, el mismo, produce contradicciones porque trabaja con una falsa imagen de la realidad.

De esta forma, el hombre vive en una forzada ignorancia porque le es imposible discernir desde la transparencia de los elementos tangibles e intangibles que componen el ambiente en que se desarrolla. Bajo ese escaso conocimiento, con una visión reducida y además distorsionada por la opacidad que posee la realidad; los sucesos que ocurren e irrumpen en la vida del hombre, lo amenazan porque no tiene las facultades para manejar la aleatoriedad de esos sucesos y provocan, en muchos casos, la ruptura de sus creaciones.

La obra del hombre es manifestación de su frágil memoria y reducido conocimiento de la realidad en que opera.

Taleb entiende la realidad como un conjunto de sistemas, donde un sistema es un vínculo entre elementos engranados para que una totalidad mayor funcione correctamente, y observó que los sistemas que componen la realidad, al enfrentar las amenazas que ella misma desata, se comportan dentro de tres paradigmas que él mismo caracteriza: el paradigma de lo frágil, el paradigma de lo robusto y el paradigma de lo antifrágil (FIGURA 1).

Dentro del primero -el paradigma de lo frágil-, un sistema no es capaz de resistir a una crisis, porque no está preparado para prevenir ni hacer
1. Esquema que representa las distintas curvas que pueden tener los sistemas al enfrentarse al desorden o crisis dependiendo en qué paradigma se encuentran. Aquellos frágiles, dan una curva negativa ya que el sistema se rompe, los robustos, no presentan cambios y se mantienen lineales ya que no se rompen pero tampoco mejoran y aquellos antifrágiles muestran una curva positiva ya que presentan mejorías en el sistema a través del beneficio del desorden. Fuente: Elaboración propia.

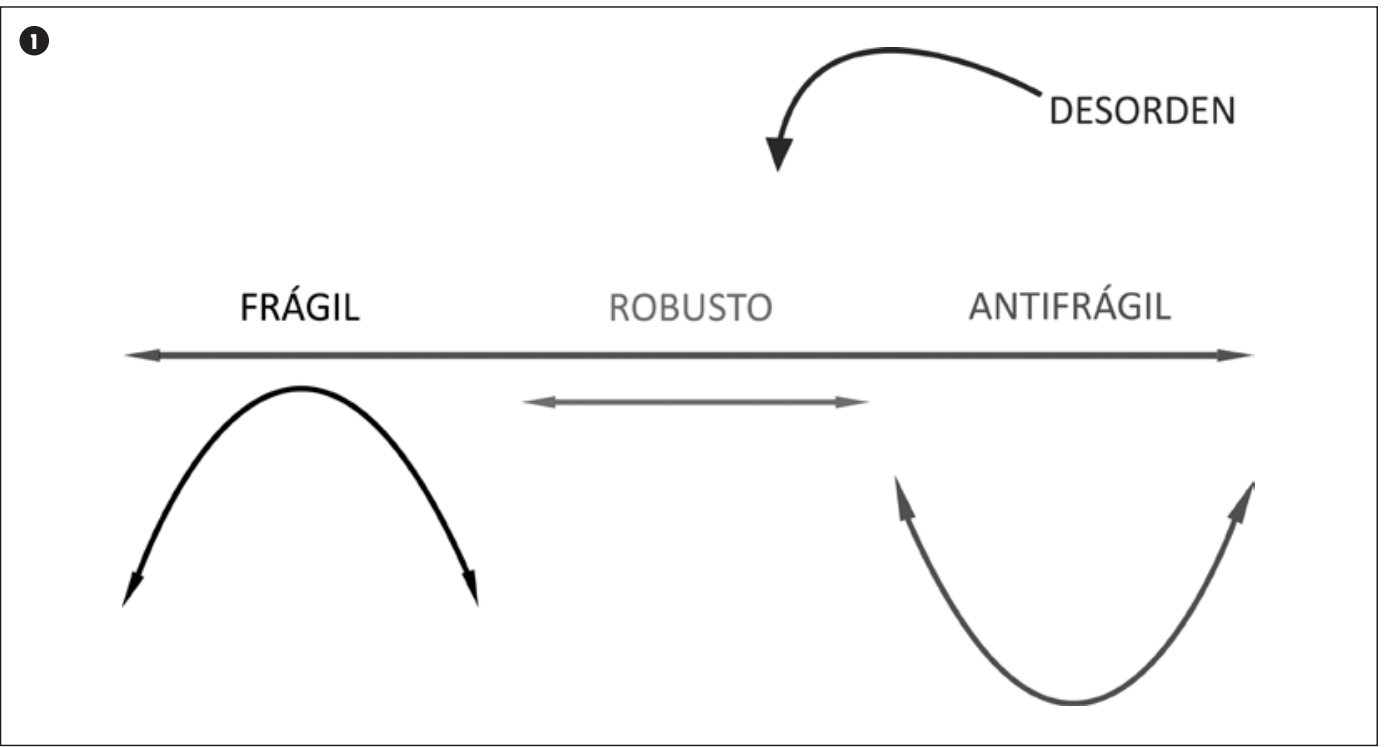

frente a ella, terminando con la ruptura del sistema. En arquitectura, los sistemas que son frágiles y tienden a la ruptura tras enfrentarse a un suceso de crisis -como lo puede ser un terremoto- han sido creados en un escenario de ignorancia y poca exploración sobre las problemáticas existentes del contexto y los futuros sucesos que pueden irrumpir en él. Se opta por la repetición de soluciones que han funcionado en otro lugar, espacio y tiempo, produciendo contradicción en el entorno y la imposible adaptación de los sistemas.

El segundo paradigma -el de lo robusto-, se refiere a la capacidad que tienen los sistemas de resistir, permaneciendo en un estado deseable tras una crisis importante. No se rompe, pero tampoco se produce una mejoría del sistema; simplemente sobrevive, se mantiene en un estado de normalidad. Los sistemas robustos dentro de la arquitectura, según el estudio que se realizó sobre la teoría del arquitecto Stan Allen, quien plantea el inicio de la búsqueda de la real demanda del contexto en que se insertará el sistema, a través de la reinvención de las herramientas que se utilizan para estudiar y proyectar en un entorno incorporando soluciones innovadoras. El sistema es capaz de proyectarse dentro de su entorno y las transformaciones que este puede tener con el tiempo, otorgándole la capacidad de resistir a situaciones adversas. Pero con el paso del tiempo terminará insoslayablemente por romperse.

En el tercer paradigma -el de lo antifrágil-, los sistemas se crean y actúan de la misma forma en que lo hace la naturaleza; ejemplo por excelencia de antifragilidad. A través del manejo de la opcionalidad, la naturaleza crea sistemas que no solamente sobreviven, sino que son capaces de evolucionar incluso tras una crisis importante. De hecho, los sistemas antifrágiles se benefician de las crisis, ya que pueden aprender de ellas y, a la vez, reinventarse para mejorar. Poseen capacidades regenerativas que existen desde la creación del sistema porque se anteponen a las dificultades que puedan enfrentar a lo largo de su vida y las consecuencias que pueden tener para la integridad del sistema y la relación con su entorno. Además, a medida que sobreviven, se 
autoexponen a pequeñas dosis de crisis para ver el comportamiento que tienen y mejorar antes de que la crisis real se presente.

"La antifragilidad es más que resiliencia o robustez, lo resiliente aguanta los choques y sigue igual; lo antifrágil mejora" (Taleb, 2013, p. 25).

El problema que se diagnosticó en la investigación realizada en el seminario, es que en arquitectura, la mayoría de los sistemas que se crean y que funcionan hoy en la ciudad, se mueven dentro del paradigma de lo frágil. En Chile, han habido sismos de gran intensidad que han exigido a los sistemas que conforman las ciudades más de lo que pueden resistir $y$, muchas veces, han sufrido rupturas irreparables. Si bien, es un país que ha enfrentado bien aquel tipo de sucesos, moviéndose entre los paradigmas de lo frágil y de lo robusto, no existen garantías de que los sistemas -es decir, las estructuras que deben resistir al sismo como edificios, plazas y todo los que conforme la zona urbana- hayan presentado mejorías.

Así, la pregunta que se quiso responder en la investigación fue si era posible o no, que los sistemas en arquitectura, comenzaran a moverse hacia el paradigma de lo antifrágil y pudieran evolucionar, por sí solos, a pesar de ser sistemas inertes, como lo hacen los sistemas en la naturaleza.

"Y es que la idea de la antifragilidad no forma parte de nuestra conciencia, pero, afortunadamente, sí que forma parte de nuestra conducta ancestral y nuestro equipo biológico, y es una propiedad presente en todos los sistemas que han sobrevivido" (Taleb, 2013, p. 55).

La investigación comenzó por estudiar la teoría de Taleb en el mundo de la economía y la bibliografía que ella ofrecía, que fundamentalmente estaba relacionada con investigaciones científicas del área de la medicina, teoría de sistemas y manejo de riesgos. Luego, para establecer un nexo con el mundo de la arquitectura, se comenzaron a buscar teorías que al menos se movieran en el paradigma de lo robusto y fue así como se llegó a estudiar la teoría de Stan Allen.

Allen, además de ofrecer una teoría que fortalece la coherencia en arquitectura y restablece la relación con el entorno en que se insertan los proyectos, es un autor que trabaja en la esfera teórica para luego aplicar en la práctica y representación de la arquitectura sus postulados, que es precisamente lo que se buscaba personalmente en la investigación que se estaba realizando; proponer una teoría para que luego se aplicara en la práctica.

"La práctica descubrirá nuevos usos de la teoría solo si se mueve cerca de la compleja y problemática realidad" (Allen, 2000, p. 17).

La teoría que plantea Taleb es precisamente una que se mueve muy cerca de la "compleja y problemática realidad", ya que la estudia desde cerca y propone una estrategia que imita la conducta ancestral de la naturaleza que observó en los sistemas orgánicos.

La base teórica, conformada por Taleb y Allen, definió el marco teórico que se desarrolló en el seminario para describir la complejidad de la realidad, la característica distorsión que ella tiene para el hombre debido a la enorme cantidad de información y poca claridad con que se presenta, el fenómeno de la aleatoriedad que poseen los sucesos y, cómo manejar los daños que ellos pueden provocar en los sistemas que se crean. A medida que se iba describiendo el marco teórico, se llegó a la conclusión de que todo lo que compone la realidad, es información. Puede estar en orden o desorden y se puede percibir caóticamente o como una complejidad organizada, pero siempre será la misma información, toda es relevante y, lo más importante, es que puede arrojar la probabilidad de que una crisis se presente.
Una vez desarrollado el marco teórico, se inició el estudio de los ejemplos que el propio Taleb planteó en su teoría sobre urbanismo y arquitectura frágil y antifrágil. Se decidió estudiar aquellos ejemplos que el autor del concepto planteó, ya que la investigación que se estaba realizando era el primer acercamiento teórico de la antifragilidad en arquitectura, por lo que era necesario entender por qué postulaba a esos ejemplos y qué información arrojaban. Los ejemplos analizados fueron Le Corbusier y Robert Moses catalogados como frágiles en urbanismo, Jane Jacobs como antifrágil en urbanismo y Antoni Gaudí como antifrágil en arquitectura.

Los dos primeros, son similares para Taleb ya que ambos privilegiaron en su obra al automóvil antes que a los propios habitantes, poseían métodos de diseño que los alejaban completamente de la compleja realidad porque rechazaban el caos y el desorden que identificaban en las ciudades. Moses diseñaba a partir de fotografías aéreas y, en el caso de Le Corbusier, eliminaba lo existente para trabajar en un terreno llano e imponer el orden que él mismo definía. En cambio, Jacobs, plantea una teoría de urbanismo basada en la experiencia en tiempo real con el sitio en que se trabaja e interpreta el desorden de la ciudad como una complejidad organizada y valora la riqueza de ello y lo explota con el fin de generar diversidad en la ciudad. Gaudí, es catalogado como antifrágil porque fue un arquitecto que se inspiró en la naturaleza al igual que la teoría de la antifragilidad de Taleb, y se esforzó por entender la complejidad de los materiales con que trabajó a través de la constante exploración para definir la estructura y cada uno de los elementos que conformaban sus construcciones.

Se escogieron de cada ejemplo, dos o más obras para estudiar las teorías y propuestas que cada uno planteaba y posteriormente analizarlos bajo cuatro criterios que se fueron definiendo a medida que iba avanzando la investigación. Cada criterio tiene su origen en diversos autores, 
el primero, en la teoría de Stan Allen, el segundo en la experiencia personal que se tuvo al visitar la Bienal de Venecia el año 2014, el tercero en el estudio del trabajo exhaustivo de Gaudí y el último en la teoría de Taleb y de algunos artículos que se estudiaron sobre investigaciones científicas para medicina. Los criterios se pueden definir de la siguiente forma:

- "Desde abajo hacia arriba", que plantea una aproximación al contexto que inicie desde lo que existe, pase por lo que se necesita buscando la real demanda del entorno, incorporando lo que se quiere diseñar y se proyecte hacia las futuras transformaciones que pueda sufrir, para aclarar las opacidades que posee la realidad y trabajar con una real imagen del contexto.

- "Tiempo, espacio y existencia", son variables que se deben incorporar a la creación de sistemas porque determinan la permanencia en el tiempo de un espacio y su capacidad de evolucionar en conjunto con las diversas existencias y, para que ello ocurra, el espacio debe ofrecer algo concordante con la necesidad del tiempo y las mutaciones el desate en lo diseñado.

- "Creación inventiva", es la forma de producir innovación en la creación de sistemas que promueva la adaptación de ellos y sean capaces de crear nuevas formas de sobrevivir dependiendo del tipo de crisis a la que se enfrenten, a través de la reinvención de las herramientas y la exploración con las formas y materiales que ofrece la propia realidad en conjunto con la creación genial del hombre.

- "Visión orgánica", es precisamente una visión que se debe tener para enfrentar un proceso creativo de diseño donde se incorpore la mayor cantidad de probabilidades y variables al construir un sistema, para tener un bagaje grande de opciones y poder elegir la mejor opción, como lo hace la naturaleza.
En el desarrollo del seminario se analizaron todos los ejemplos bajo cada criterio, priorizando la explicación de estos últimos por sobre las teorías de los autores para que en un futuro se pudieran analizar otros casos. El análisis realizado se basó en la recopilación de propuestas y obras a través de material gráfico y citas de cada autor que reflejaran conductas frágiles, robustas o antifrágiles con el fin de catalogar a cada ejemplo en un paradigma específico.

Sobre los casos de Le Corbusier y Robert Moses, se obtuvo casi la misma información debido a la similitud que existía en ambos, se catalogaron en los criterios "desde abajo hacia arriba" y "visión orgánica" como frágiles y, en los criterios "tiempo, espacio y existencia" y "creación inventiva" como robustos ya que, a pesar de que existiera innovación en sus obras y que hayan perdurado a través del tiempo, no son sistemas evolutivos, más bien rígidos y con fundamentos impuestos que no surgieron de la lectura del entorno.

El caso de Jane Jacobs, a diferencia de los otros ejemplos mencionados, demostró cualidades antifrágiles en todos los criterios de análisis, su planteamiento teórico es catalogado por Taleb y también por el análisis realizado, como un pensamiento antifrágil. Principalmente por el entendimiento orgánico de un sistema inerte y la aproximación a la vida urbana desde la experiencia personal, la propuesta de generadores de diversidad para la ciudad como un método que garantiza la adaptabilidad y versatilidad del espacio a través del tiempo.

Por último, el caso de Antoni Gaudí, fue el que ofreció el mayor acercamiento antifrágil a la etapa proyectual de la arquitectura y no solamente en la esfera teórica. Su creación genial y su afán por explorar constantemente y reinventar casi diariamente cada elemento que era parte de una obra lo caracterizó en crear obras que estuvieran lo mejor adaptadas posible a la demanda de su tiempo. Entendió la arquitectura como una realidad viva y le otorgó de una organicidad que hizo posible estructuras impensadas para su época y que hasta el día de hoy sorprenden a la ignorancia del hombre contemporáneo. Su genialidad es inminente y ha trascendido a lo largo del tiempo gracias a la capacidad que tuvo Gaudí de darle versatilidad a sus obras para que pudiera no solo resistir a sucesos aleatorios del tipo natural, sino que, generó una invención nueva y diversa para cada caso particular entendiendo el contexto y cómo ese se proyectaba hacia el futuro. Al ofrecer un nuevo conocimiento, fue capaz de generar una evolución no solo en sus obras, sino que en toda la esfera de la arquitectura.

El análisis que se realizó, concluyó con que la hipótesis de la investigación, no había sido comprobada, ya que los sistemas que se analizaron, catalogados como antifrágiles, no demostraron que esas conductas surgieran del propio sistema como es el caso de los sistemas que crea la naturaleza, sino que, por el contrario, todas las competencias que pueda tener un sistema arquitectónico de carácter antifrágil, están dadas por el hombre.

En el caso de Gaudí, las capacidades estructurales de sus obras poseen organicidad y dan la sensación de que fueran parte de su entorno porque fue él quien dedicó una vida entera a forjar aquello, fue su innovación y estudio exhaustivo lo que permitió lo que Taleb llama antifragilidad a través de la fractalidad en sus obras. El fractal es una estructura que está conformada por un patrón que se repite y que es legible tanto en sus partes como en la forma total de la estructura, es un método matemático para construir sistemas y Gaudí lo utilizó imitando la naturaleza que posee muchas entidades fractales. Así, lo que ofrece Gaudí, es un método genial de creación de sistemas que le otorgó en ese tiempo, rasgos antifrágiles a sus obras. 
El hombre es el agente evolutivo, es el ejecutor de innovaciones y creador de sistemas que pueden o no ser antifrágiles. Depende de su voluntad y de su visión si el propósito antifrágil se toma en cuenta o no para enfrentar los sucesos que someten a los sistemas hoy en día. En el caso de la arquitectura, los sistemas son pensados, diseñados y construidos por el hombre -o al menos así fue el caso de la arquitectura de Gaudí, quien participó y gestó sus obras desde el proyecto hasta la construcción-y es responsabilidad de él, dar las competencias necesarias para que se puedan comportar de manera antifrágil al enfrentar un suceso aleatorio.

La antifragilidad es un desafío para el hombre que habita la compleja realidad y está en su capacidad creativa y de innovación la oportunidad de dar soluciones antifrágiles a las obras que diseña.

\section{REFERENCIAS}

Allen, S. (2000). Practice: Architecture, tecnique and representation (Critical voices in art, theory and culture 1. Architecture 2. Representation (Phylosophy) I. Title II. Agrest, Diana. Amsterdam, The Netherlands: G+B Arts International Imprint.

Taleb, N. (2013). Antifrágil: Las cosas que se benefician del desorden. Buenos Aires, Argentina: Paidós.

Munjin, K. (2015). Antifragilidad en la arquitectura: Sistema orgánico para resistir el desorden y evolucionar. Seminario de Investigación, Instituto de Historia y Patrimonio, Universidad de Chile. 